\title{
WN segmentation of setina images for the early diagnosis of Alzheimer's disease (AD)
}

\begin{abstract}
Alzheimer's disease (AD) is a common form of senile dementia. Although our understanding of the key steps underlying neurodegeneration in Alzheimer's disease (AD) is incomplete, it is clear that it begins long before symptoms are noticed by patient. Conventional clinical decision making systems are more manual in nature and ultimate conclusion in terms of exact diagnosis is remote. In this case, the employment of advanced Biomedical Engineering Technology will definitely helpful for making diagnosis. Any disease modifying treatments which are developed are most possibly to be achieving success if initiated early in the process, and this needs that we tend to develop reliable, validated and economical ways to diagnose Alzheimer's kind pathology. However, despite comprehensive searches, no single test has shown adequate sensitivity and specificity, and it is likely that a combination will be needed. Profiling of human body parameter using computers can be utilised for the early diagnosis of Alzheimer's disease. There are several imaging techniques used in clinical practice for the diagnosis of Alzheimer's type pathology. There are lot of tests and neuroimaging modalities to be performed for an effective diagnosis of the disease. Prominent of them are Magnetic Resonance Imaging Scan (MRI), Positron Emission Tomography (PET), Single Photon Emission CT Scanning (SPECT), Fundus Imaging and Optical Coherence Tomography (OCT). In the recent studies made on Alzheimer's disease it is clearly investigated that are some parameter changes on the retina of the eye of the AD patients. In this research we have proposed a new scheme based on Wavelet Networks (WN) for the segmentation of fundus retinal images for the early diagnosis of AD.
\end{abstract}

Keywords: alzheimer disease; dementia, wavelet networks (WN), sombrero, fundus image, early diagnosis
Volume 7 Issue 2 - 2018

\author{
Sandeep CS, Sukesh Kumar A \\ Department of ECE, University of Kerala, India
}

Correspondence: Sandeep CS, Department of ECE, College of Engineering, University of Kerala, Trivandrum, India, Email sandeepcs07nta@gmail.com

Received: November 18, 2017 | Published: April 09, 2018
Abbreviations: AD, alzheimer's disease; MRI, magnetic resonance imaging; PET, positron emission tomography; SPECT, single photon emission CT; OCT, optical coherence tomography; MCI, mild cognitive impairment; APP, amyloid precursor protein; $\mathrm{CT}$, computed tomography; ANN, artificial neural network; FDG, fluorodeoxyglucose; MLP, multilayer perceptrons; RBF, radial basis functions; FGWNs, fixed-grid wavelet networks; BP, back propagation; OLS, orthogonal least square

\section{Introduction}

Alzheimer's disease (AD) is a progressive deteriorating and loss function of the neurons in the human brain and leads to changes in the retina region. It leads to loss of memory of the subject and weakens the proficiency in doing a sequence of actions regularly following. ${ }^{1-5} \mathrm{AD}$ is the sixth-leading cause of death among various diseases and is $70 \%$ widespread in all cases of dementia. ${ }^{6}$ According to another report every $71 \mathrm{sec}$, someone develops Alzheimer's disease and the rate doubles roughly every 10 years after age $65 .^{7}$ Some studies show that almost 36 million people are believed to be living with Alzheimer's disease and other types of dementias. This will increase to about 66 million by 2030 and nearly 115 million by $2050 .^{8}$ The development of AD can be placed into four stages. The first stage is called Mild Cognitive Impairment (MCI) that does not make prominent changes in day to day living. The second and third stages of the disease are called as Mild and Moderate AD. These stages describe the distinctive nature by a rise in cognitive shortfall, and reduction in independence. The fourth stage is called Severe $\mathrm{AD}$ in which the affected person almost dependent on caregivers and an overall decline of personality. ${ }^{9}$ Alzheimer's disease is one of the underlying causes of dementia, the term used to point out weakened brain functions and related symptoms like difficulty in performing routine tasks, memory loss, confusion, loss of intellectual functions and poor judgment. The above mentioned conditions are similar symptoms of below mentioned neurological disarrays. This includes Alzheimer's disease, Frontotemporal Dementia, vascular dementia, Dementia with Lewy Bodies and Parkinson's disease. AD is the most common type of dementia and is clinically evident when there is gradual loss of brain functions. The symptoms thus occurring may lead to disorientation and aphasia (difficulty in language), indicating cortical dysfunction, agnosia (impairment in recognizing object and people), apraxia (impaired motor function) and significant of all, memory impairment. As the disease develops drastically, the patients suffer disability and immobility. The brain of such patients shows gross cortical atrophy with ventricular enlargement. The most widely known neuropathological hallmarks of $\mathrm{AD}$ are senile plaques which are seen outside the neuron and neurofibrillary tangles that are seen inside the neuron. Neurofibrillary tangles are filamentous bundles in cytoplasm of the neurons displacing or encompassing nucleus. In the pyramidal cells, they appear as 'flame' while in rounder cells they appear as 'globos tangles'. ${ }^{10}$ Senile plaques present outside the neuron, appear as spherical bodies bearing dilated and tortuous neuritic processes around an amyloid beta core which contains some abnormal proteins like amyloid beta plaques which are derived through the processing of Amyloid Precursor Protein (APP). ${ }^{10,11}$ Familial causes or genetic reasons involved in disease pathology include mutations on chromosomes 21, 14 and 1. Risk 
factors for AD are elder age, small head size, history of head trauma, lower intelligence, and female gender. ${ }^{12,13}$ The imaging modalities tests that were established for AD are Computed Tomography (CT), Magnetic Resonance Imaging (MRI), Positron Emission Tomography and neuropsychological tests. CT scans were used to check for structural deterioration of the brain and increased ventricle size. It was noticed that at firstly cerebral atrophy was predominant in AD patients than control subject. However it was discovered later that healthy people also have cerebral atrophy. Patients with dementia may not have cerebral atrophy at least in the early stages of the disease. From these findings it was difficult to distinguish between a healthy elderly patient and a patient with dementia. So the CT scans have been deemed as clinically not as useful in the primary diagnosis of AD. After CT structural MRI was introduced to evaluate MCI (mild cognitive impairment) to AD in addition to clinical measures. Structural MRI measures the whole brain volumes, medial temporal lobe structures, and ventricular volumes. Therefore MRI can be helpful in differentiating between $\mathrm{MCI}$ and $\mathrm{AD} .{ }^{14} \mathrm{PET}$ is an imaging modality that uses biochemical ways of getting images rather than structural information. Alzheimer's disease is one of the underlying causes of dementia. Dementia is the term used to indicate impaired brain functions and encompass symptoms like memory loss, confusion, difficulty in performing routine tasks, loss of intellectual functions and impaired judgment. PET technology includes the detection of photons which records the levels of radioactivity beginning from given points in time and space. Positron emitting radioisotopes are used to generate the radioactivity. ${ }^{15}$ PET scan measures different compounds in the brain especially the fluorodeoxyglucose (FDG) that can compete with glucose for metabolism and absorption in neurons. With AD the neurons intake of glucose and FDG becomes less. By projecting the regions of decreased FDG uptake, PET can help in the early diagnosis of $\mathrm{AD}$, even in the absence of the gross structural damage detected by other imaging techniques such as $\mathrm{CT}$ or magnetic resonance imaging. ${ }^{16}$ Some studies have been conducted to examine patients that are amyloid positive or amyloid negative, PET has been used extensively to study $\mathrm{AD}$, and it is evolving into an effective tool for early diagnosis. PET is a very costly scan to perform the test for AD, it has been the most useful to provide visual images in the detection of the disease. There are some recent advances in technology that can not only detect $\mathrm{AD}$, but it can possibly explain the symptoms and how the disease works. The neuropsychological tests are used to examine the specific type and level of cognitive impairment that the patient is having. Some of them that were, "Mini Mental State Examination, Trial Making Test parts A and B, Digit Symbol Substitution Test, Digit Span forward and backward, Rey Auditory Verbal Learning Test, category fluency, and the Clock Drawing task". ${ }^{13}$ All of these tests are helpful in showing the memory recall of a patient and the realizable areas where the patient may degrade. Using the above different tests, it can be helpful to determine the types of treatment plans which are to be used. However neuropsychological tests alone are not helpful in detecting early $\mathrm{AD}$, trials were often conducted combining neuropsychological tests with clinical tests and various imaging modalities. For an effective and early diagnosis of AD, a population based study is necessary and required, which gives an idea about the various tests involved in determining AD.

Recently, studies show that there are changes not only in the brain but also in the retina of Alzheimer's disease patients. ${ }^{17-19}$ For this purpose we have used the fundus images of retina of $\mathrm{AD}$ patients obtained from fundus camera for the diagnosis. The standard approach in automatic fundus image analysis consists of image acquisition, preprocessing, image segmentation, feature extraction, feature selection and classification. Image acquisition is done by fundus camera which is shown in Figure 1; it is a kind of imaging technique used to examine the different eye related disease. Preprocessing is by using median filter which removes unwanted noises on the obtained fundus image of the eye. For the different steps involved in fundus image analysis of retina, segmentation is the most important stage of all. Image segmentation is defined as the process of partitioning a digital image into multiple segments. The goal of segmentation is to simplify and/or change the representation of an image into something that is more meaningful and easier to analyze. Image segmentation is mostly used to locate objects and boundaries like lines, curves etc. in images. More accurately, image segmentation is the process of assigning a label to every pixel in a digital image such that pixels with the same label share certain visual characteristics. The result of image segmentation is a set of segments that collectively cover the whole image, or a set of contours extracted from the image. Each of the pixels in a region is similar with respect to some characteristics such as such as color, intensity, or texture.

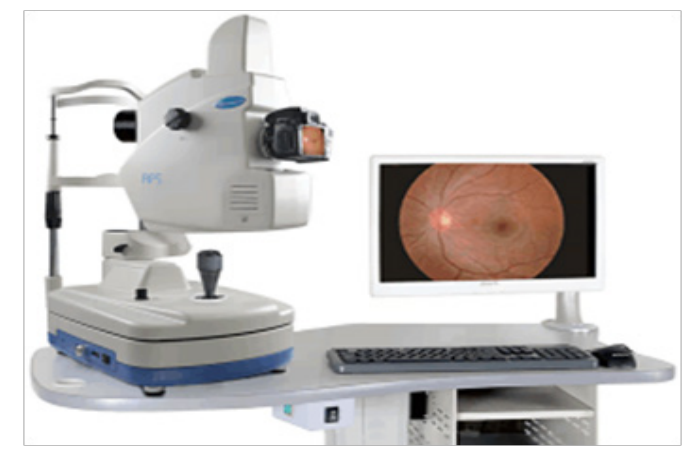

Figure I fundus camera and fundus image obtained on the screen.

There are different ways for the segmentation of images in the artificial intelligence field. Most prominent and popular of them are fuzzy logic and artificial neural network (ANN) approaches for segmentation of medical images..$^{20,21}$ Apart from above another promising computational intelligence method that has been widely used for various applications in different areas is wavelet network (WN). A wavelet can be called as a wave like oscillation with amplitude that begins at zero, increases, and then decreases back to zero. A family of wavelet scan is formed from a function, called "mother wavelet," which is confined in a finite interval. "Daughter wavelets" are then formed by shifting and scaling of mother wavelet. Wavelets are mainly useful for compressing image data from a larger one. Wavelet network takes full advantage of the characteristics of denoising, back ground reduction, and recovery of the characteristic information and Neural Network capacity of universal approximation. ${ }^{22-25}$ For this reason, it has a great ability to be used in many different applications. ${ }^{26,27}$ For instance, in image processing wavelet networks have overcome many of the limitations in other intelligent methods such as artificial Neural Networks. The main advantage of wavelet networks over similar architectures such as multilayer perceptrons (MLP) and networks of radial basis functions (RBF) is the possibility of optimizing the wavelet network structure by means of efficient deterministic construction algorithms. ${ }^{28}$ In this paper a specific wavelet network for segmentation of fundus images is employed. Wavelet networks are classified into two groups. They are adaptive wavelet networks (AWNs) and fixedgrid wavelet networks (FGWNs). ${ }^{29}$ Adaptive wavelet network is 
continuous wavelet transform whereas FGWN is discrete wavelet transform. Due to numerous shortcomings of AWNs like for example, complex calculations, sensitivity to initial values, and the problem of measuring initial values, their application is limited..$^{29}$ In an FGWN, the outer parameters of the network like number of wavelets, scale, and shift parameters value are determined. The only inner parameters of the network, weights are specified by algorithms similar to the least squares. These types of networks do not need training. In AWNs, initial values of network parameters including weights of neurons, shifts, and scales of wavelets are selected randomly or using other methods. These parameters are then updated in the training stage by means of techniques such as gradient descent or back propagation (BP).

Then, the optimized values of network parameters are calculated. But in an FGWN, the number of wavelets, as well as the scale and shift parameters, can be determined in advance and the only unknown parameters are the weight coefficients which are calculated through methods such as least squares. So in proposed FGWN, there is no need to specify random initial values for parameters or to use gradient descent, BP, or other iterative methods. Normally, in training stage of an adaptive network, all the parameters change; on contrary, in FGWN only, the weights are specified during an on iterative process. Thus, it could be concluded that FGWNs do not need training procedure. A three-layer FGWN with one hidden layer is employed Specific wavelet network or fundus image segmentation as shown in Figure 2. The procedure for image segmentation in this paper is as follows. At first the input data are normalized. Then, after selecting a proper mother wavelet, which is usually Sombrero because of its desirable characteristics such as convenient calculates on, adaptability to Gaussian structures, and robustness against noise. ${ }^{30}$ Next a wavelet lattice is formed. Wavelet lattice is a hyper shape of shift and scale values of wavelets. The huge dimensions of this hyper shape should be decreased and effective wavelets should be selected. All of these are accomplished through two stages of screening. This paper is unique as it employs two stages of screening. This gives ground to increase the popularity of the wavelet lattice and to estimate the function in a more accurate way which is most beneficial and significant for larger scales. The author's previous works in the area of Biomedical Engineering will definitely help to develop a new proposed tool using latest biomedical methods for the solution of the early diagnosis of AD. ${ }^{1-5}$ The organization of this paper is as follows: The basic concept of WN is introduced in Section II. In this section, the WN structure with the algorithm necessary for formation of network and determination of its parameters is presented. In Section III, the proposed segmentation process based on WN is introduced. The simulation results of the proposed method in comparison with other approaches are illustrated in Section IV. Finally, in Section V, a summary and conclusion of the findings of the study is presented.
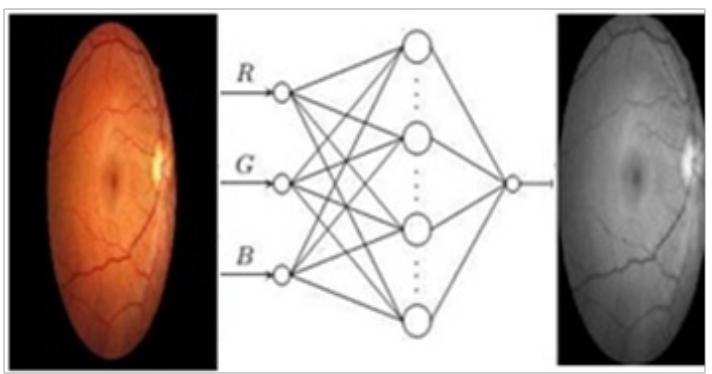

Figure 2 Presented FGWN for segmentation of fundus images.

\section{WN segmentation}

A major advantage of wavelet networks over other neural architectures is the availability of efficient construction algorithms for defining the network structure. ${ }^{31}$ In this paper we have used The FGWN structure is determined by a ten-stage algorithm such as Normalization, Selecting the mother wavelet, Choosing the scale and shift parameters, Formation of wavelet lattice, Primary screening, Secondaryscreening, Formation of wavelet matrix, Performing orthogonal least square (OLS) algorithm, Selecting the number of wavelons and Calculating wavelons weight coefficient. The algorithm mentioned above is used in the present stage for segmentation is the retinal fundus images. From images database, a number of images are randomly chosen for formation of FGWN. At first the values of R, G, and $\mathrm{B}$ matrices of each colour fundus image are mapped into $[0,1]$ range by performing normalization process. FGWN is formed with three inputs, a hidden layer, and an output. In order to form the FGWN, the values of three colour matrices are considered as network inputs. These matrices are related to the five chosen images from the selected images for segmentation. From these images, some pixels are selected randomly (ranging from 1000 to 5000 pixels for a $485 \times 716$ image). If the pixel is inside the lesion, network output will be considered asn0, and if the pixel is outside the lesion, the output will be considered as 1. In this way, the FGWN is formed. After that, the value of the three matrices R, G, and B for each pixel are considered as FGWN inputs, and the output of FGWN is a binary image that shows the segmented of original image.

After segmentation post processing is done .Since extracting the features of lesion is the most essential part of diagnosing melanoma, extracting the exact boundary of lesion is a vital task. For this, after segmentation with an FGWN the space between two shapes is filled, extra parts are eliminated, and the noise is removed. Then, the exact boundary of lesion is extracted. This is done by appropriate morphological processes, including erosion, dilation

\section{Conclusion}

In this study a new approach for the segmentation of fundus retina images based on fixed grid wavelet network (WN) has been employed. A wavelet lattice is formed. Parameters of wavelets are determined with two screening stages. Orthogonal least squares algorithm is used to calculate the network weights and to optimize the network structure using the developed algorithm, hence provides a useful tool for the analysis of fundus retina images.

\section{Acknowledgements}

None.

\section{Conflict of interest}

The author declares that there is no conflict of interest.

\section{References}

1. Sandeep CS, Sukesh Kumar A. Early Prediction of Alzheimer's Disease by Examining Changes in Eye Parameters. J Anal Pharm Res. 2017;5(6):00159.

2. Sandeep CS, Sukesh Kumar A. A Review on the Early Diagnosis of Alzheimer's Disease (AD) through Different Tests, Techniques and Databases. AMSE. 2015;76(1):1-22. 
3. Sandeep CS, Sukesh Kumar A, Susanth MJ. The Online Datasets Used to Classify the Different Stages for the Early Diagnosis of Alzheimer's Disease (AD). International Journal of Engineering and Advanced Technology. 2017;6(4):38-45.

4. Sandeep CS, Sukesh Kumar A. A Psychometric Assessment Method for the Early Diagnosis of Alzheimer's disease. International Journal of Scientific \& Engineering Research. 2017;8(3):901-905.

5. Sandeep CS, Sukesh Kumar A, Susanth MJ. The Early Diagnosis of Alzheimer Disease (AD) Using CAMD, TREAD and NAAC Databases. International Journal for Science and Advance Research In Technology. 2017;3(3):366-371.

6. Sandeep CS, Sukesh Kumar A. A Review Paper on the Early Diagnosis of Alzheimer's Disease (AD) through Profiling of Human Body Parameters. International Journal of Computer Science and Engineering Communications. 2013;1(1):21-29.

7. Alzheimer's Facts and Figures. Alzheimer's Association.

8. http://www.alz.co.uk/media/nr100921.html

9. Frosch MP, Anthony DC, Girolami UD. The Central Nervous System. In Robbins and Cotran Pathologic Basis of Disease. In: Robbins SL, Kumar V, et al. editors. Philadelphia: Elsevier; 2010:1313-1317.

10. Harvey RA, Champe PC, Fisher BD. Lippincott's Illustrated Reviews. Microbiology. 2nd ed. USA; Lippincott Williams and Wilkins. 2006:432.

11. Cummings JL, Vinters HV, Cole GM, et al. Alzheimer's disease: etiologies, pathophysiology, cognitive reserve and treatment opportunities. Neurology. 1998;51(1):2-17.

12. Yaari R, Corey-Bloom J. Alzheimer's disease: Pathology and pathophysiology. Semin Neurol. 2007;27:32-41.

13. Larson EB, Wang L, Bowen JD, et al. Exercise is associated with reduced risk for incident dementia among persons 65 years of age and older. Ann Intern Med. 2006;144(2):73-81.

14. Mayeux R. Epidemiology of neurodegeneration. Annu Rev Neurosci. 2003;26:81-104

15. Harvey RJ, Skelton-Robinson M, Rossor MN. The prevalence and causes of dementia in people under the age of 65 years. J Neurol Neurosurg Psychiatry. 2003;74:1206-1209.

16. Chu LW, Tam S, Wong RL, et al. Bioavailable testosterone predicts a lower risk of Alzheimer's disease in older men. J Alzheimers Dis. 2010;21(4):1335-1345.
17. Kesler A, Vakhapova V, Korczyn AD, et al. Retinal thickness in patients with mild cognitive impairment and Alzheimer's disease. Clin Neurol Neurosurg. 2011;113(7):523-526.

18. Frost S, Martins RN, Kanagasingam Y. Ocular biomarker for early detection of Alzheimer's disease. J Alzheimers Dis. 2010;22(1):1-16.

19. Ohno-Matsu K. Parallel findings in age-related macular degeneration and Alzheimer's disease. Prog Retin Eye Res. 2011;30(4):217-238.

20. Cheng KS, Lin JS, Mao CW. Techniques and comparative analysis of neural network systems and fuzzy systems in medical image segmentation. Fuzzy Theor Syst Tech Appl. 1999;3:973-1008.

21. Jiang J, Trundle P, Ren J. Medical image analysis with artificial neural networks. Computerized Medical Imaging and Graphics. 2010;34(8):617-631.

22. Balabin RM, Safieva RZ, Lomakina EI. Wavelet neural network (WNN) approach for calibration model building based on gasoline near infrared (NIR) spectra. Chemometrics and Intelligent Laboratory Systems. 2008;93(1):58-62.

23. Zhang Q, Benveniste A. Wavelet networks. IEEE Trans Neural Netw. 1992;3(6):889-898.

24. Pati YC, Krishnaprasad PS. Analysis and synthesis of feedforward neural networks using discrete affine wavelet transformations. IEEE Trans Neur Netw. 1992;4(1):73-85.

25. Szu HH, Telfer BA, Kadambe SL. Neural network adaptive wavelets for signal representation and classification. Opt Eng.1992;31(9):1907-1916.

26. Zhang H, Zhang B, Huang W, et al. Gabor wavelet associative memory for face recognition. IEEE Trans Neural Netw. 2005;16(1):275-278.

27. Jemai O, Zaied M, Amar CB, et al. Pyramidal hybrid approach: Wavelet network with OLS algorithm-based image classification. Int J Wavel Multir Inf Process. 2011;9(1):111-130.

28. Galvao R, Becerra VM, Calado MF. Linear-wavelet networks. Int J Appl Math Comput Sci. 2004;14(2):221-232.

29. Billings SA, Wei HL. A new class of wavelet networks for nonlinear system identification. IEEE Trans Neural Netw. 2005;16(4):862-874.

30. Gonzalez-Nuevo J, Argueso F, Lopez-Caniego M, et al. The mexican hat wavelet family. Application to point source detection in CMB maps. Mon Not Roy Astron Soc. 2006;369:1603-1610.

31. Oussar Y, Dreyfus G. Initialization by selection for wavelet network training. Neurocomputing. 2000;34(1):131-143. 